\title{
A161 HISTOPATHOLOGICAL MODIFICATIONS IN HAEMOPHILIC SYNOVIAL TISSUE
}

L Ibba-Manneschi, ${ }^{1}$ D Melchiorre, ${ }^{2}$ A F Milia, ${ }^{2}$ M Manetti, ${ }^{1}$ G Benelli, ${ }^{1}$ E Romano, ${ }^{2}$ S Guiducci, ${ }^{2}$ S Linari, ${ }^{3}$ M Morfini, ${ }^{3}$ M Innocenti, ${ }^{4}$ M Matucci-Cerinic ${ }^{2}$ University of Florence, Italy

\subsection{6/ard.2010.129650|}

Background Haemophilia is characterised by spontaneous bleeding, mainly in the larger joints. Repeated bleeding episodes lead to synovitis and then to haemophilic arthropathy (HA) with synovial changes, cartilage damage and bone remodelling which result in disability. The pathogenesis of this blood-induced cartilage damage is very little known, but it has been suggested that the recurrent presence of blood induces synoviocyte hypertrophy and hyperplasia with intense neovascularisation and inflammation of the synovial membrane. This results in the production of catabolic enzymes and cytokines by inflammatory cells which induce and exacerbate the pathological process.

Objectives To investigate the expression in HA synovial tissue of pro-apoptotic molecules (Fas/Fas-L pathway) and molecules involved in bone remodelling (RANK/RANKL/OPG system).

Methods 20 synovial biopsies from patients with HA obtained during arthroplasty of the knee and 8 post-traumatic healthy subjects were routinely processed for light microscopic examination. The sections obtained were stained with haematoxylin and eosin to evaluate pathological changes. In order to detect in situ cell apoptosis the TUNEL method was performed. The expression of pro-apoptotic molecules Fas/FasL was examined by immunohistochemical analysis. Activation of bone remodelling was examined by analysing the expression of RANK/ RANK-L/OPG. Levels of soluble Fas and FasL, soluble RANKL and OPG in serum from 35 patients with HA and 30 healthy controls were measured by ELISA assay.

Results Microscopically, the synovium of patients with HA showed a large amount of intra- and extracellular haemosiderin deposits. The lining layer showed mild proliferation and, in the sublining area, the vessels were characterised by a thickened wall due to local and chronic inflammatory stimuli. TUNEL assay revealed a large number of apoptotic cells in the lining and sublining layers in HA patients. Moreover, TUNELpositive endothelial cells were detected. In patients with HA, Fas was highly expressed in lining layer cells and in the sublining area of the synovium. In the synovium of patients with HA, FasL positivity was observed in cells of the lining layer. Strong staining for Fas was widespread in the vessel wall, whereas mild reactivity for FasL was detected only in some vessels. A low expression of RANKL and OPG was found in HA synovium compared with controls. Serum levels of Fas were significantly higher in HA patients than in controls, while FasL levels were not different between HA patients and controls. Levels of RANKL and OPG were lower than in controls.

Conclusions Our preliminary study show that the synovial cells and vascular components in patients with HA undergo apoptosis through a Fas/FasL-mediated mechanism. In particular, Fas was strongly expressed in the synovial and vascular components, whereas FasL was not upregulated. RANKL and OPG, which compete for the same receptor (RANK), were both downregulated, suggesting a minor involvement of the RANK-mediated osteoclastogenesis in these patients. Immunohistochemical results correlate with serum levels of these proteins in patients with HA. 